\title{
Adaptive User Experience Based on Detecting User Perplexity
}

\author{
Anas Samara \\ School of Computing \\ Ulster University, Belfast \\ BT37 0QB, UK \\ samara-a@ulster.ac.uk
}

\author{
Raymond Bond \\ School of Computing \\ Ulster University, Belfast \\ BT37 0QB, UK \\ rb.bond@ulster.ac.uk
}

\author{
Hui Wang \\ School of Computing \\ Ulster University, Belfast \\ BT37 0QB, UK \\ h.wang@ulster.ac.uk
}

\author{
Leo Galway \\ School of Computing \\ Ulster University, Belfast \\ BT37 0QB, UK \\ I.galway@ulster.ac.uk
}

\begin{abstract}
As humans, our abilities and performance while carrying out different tasks differ due to several factors, such as health conditions, mental processing capabilities, emotional feelings, task difficulty and the desire to achieve it. Additionally, understanding human feelings and states that may entail information about achieving certain tasks can be an intricate job. Subsequently, this paper aims to outline the main aspects of self-ware systems and adaptive Human-Computer Interaction styles. There is an opportunity to enable machines to be more perceptive devices that can recognise innate human factors, which could be used to assist and improve the human's performance and effectiveness. This paper shows use-case scenarios of intelligent and affect-aware systems as well as presenting a conceptual model for User Perplexity as an important aspect an adaptive system should be able to capture to instantiate appropriate adaptation to the user experience. We argue that there is a subtle difference between adaptive user interfaces and adaptive user experiences.
\end{abstract}

Human-Computer Interaction, Adaptive, User Experience, Affective State, User Perplexity

\section{INTRODUCTION}

Intelligent systems are one of the most important aspects of the success of computerised services. Subsequently, designing and building a system that is capable of adaptive Human-Computer Interaction $(\mathrm{HCl})$ and 'digital' empathy similar to that in humanhuman interaction would facilitate more user task completions and user engagement. Furthermore, adaptive $\mathrm{HCl}$ requires automated monitoring of the emotional affective states of users by perceiving these states in real time. Therefore, the requirements to make an adaptive $\mathrm{HCl}$ requires information about the task along with non-verbal information from the user in real-time in order to keep the user in a positive affective state.

In the context of $\mathrm{HCl}$, the detection of the user's emotion could be just as important for adaptation and intelligent interaction with the next generation of machines (Picard, 2000). Within the domain of Affective Computing, there is no agreement on a definitive set of human emotions to recognise. Hence, several research efforts have defined different sets of emotion labels and taxonomies that are domain and application specific. For example, Ekman and Friesen (Ekman and Friesen, 1971) revealed six emotions that can be inferred from facial expressions, including surprise, fear, happiness, sadness, anger and disgust. Whereas in Shan et al. (Shan, Gong and McOwan, 2007), a different set of emotions were detected from body gestures, including anger, anxiety, boredom, disgust, joy, puzzlement and surprise. By contrast, in Busso et al. (Busso et al., 2004), only four emotions were identified, which included sadness, anger and happiness, along with a neutral state. Consequently, several studies focused on detecting different sets of emotions.

Furthermore, the idea of enhancing the interaction style between humans and computers has evolved in many ways and in different directions. With the emergence of Affective Computing, there has been research that has focused more on enhancing $\mathrm{HCl}$ by imitating human-human interaction styles, which is characterised as being empathetic whilst supporting effective communication via conversation space (Picard, 2000; Harper et al., 2008). In addition, this work becomes focused toward user engagement and production of a more attractive User Experience (UX) out of intelligent computer software that able to interpret actual affective states (Karray et al., 2008; O'Brien and Toms, 2008).

Consequently, the aim of current paper is to describe the main aspects of self-aware systems and adaptive form of $\mathrm{HCl}$. The subsequent section herein will discuss two use case scenarios of selfaware systems. Further details are discussed regarding the user's affective states and human 
feelings. Moreover, the concept of User Perplexity and detecting the moments when a user is perplexed whilst he/she is attempting a computerised task in order to facilitate adaptation is also explained. Arguably, user confusion or perplexity is a key affective state to be avoided when interacting with a system (Liu and Baker, 2013).

\section{AFFECT-AWARE SYSTEMS}

Two case scenarios are given in Figure 1 and Figure 2. These scenarios demonstrate two different cases of affect-aware systems, where the systems infer and utilise the user's feelings, and attempt to foster the completion of the desired task that they are trying to accomplish. The two systems are completely different in the services they provide, and the functionality they afford and serve. However, the pivotal component that should exist in any intelligent system should perceive non-verbal cues and understand affective states of the user within that contextual scenario, which provides a key aspect of adaptive $\mathrm{HCl}$ or indeed adaptive UX.

Scenario presented in Figure 1 involves an older man who is digitally illiterate and has no experience in using computer interfaces. He needs to interact with a computer interface in order to self-check-in and he could not avoid this check-in process. Also, he was confused, and no staff was nearby to help. Therefore, an affect-aware machine would be very helpful in this scenario. Even though this system does not update or apply any kind of adaptation in the interface itself; it still recognises the difficulty that the user had faced and automatically prompts the help desk to assist the user in completing the task.

\footnotetext{
Joe Bloggs is a 56 years old business man, who works for ACME LTD as sales executive. He decided to travel for leisure to Hawaii. His friend John helped him and prepared the booking for the hotel and the flight ticket. Joe arrived at the airport before 1 hour to his flight departure, and he wanted to check-in for his luggage, he loaded the luggage inside the cart, and put his ticket under the reader that uses infrared technology, the screen of this machine showed that the check-in process cannot be completed and beneath this message a line with red font "click here for details". However, Joe has no experience in dealing with such interfaces; he misunderstood what was going on. He unloaded the cart and put them back again, and he could not proceed. Joe started getting frustrated and angry. Fortunately, this ticket reader machine was supported with eye tracker and camera technology and the machine predicted Joe's feelings of perplexity and pinged the airport office about a problem, and announced: "We are sorry for disturbing you, Please wait a moment. Our help desk advisor on her way to you".
}

Figure 1: Use case scenario of an Intelligent check-in machine.

On the other hand, the scenario presented in Figure 2 involves a slightly different kind of an intelligent computer software that can predict user confusion with the input of an unobtrusive eye tracker fixed above the monitor. This sort of system is very helpful for users and increases the chance to enable them to complete their task efficiently and effectively. This is important since many users give up a task due to getting stuck in a counter-intuitive stage of the interaction.

George Burdell is a student who officially was enrolled in a suit of two e-learning training modules in management skills and leadership integrity at Georgia Tech. The modules are interactive wizards, which give a piece of information about a single concept, then make simple review using multiple choice and drag-and-drop questions. George began the reading part, and he intended to proceed to the next concept, but he got the review part on the screen. In this moment, George was not aware what he should do. Fortunately, an eye tracker was fixed above the monitor; and it detected his pupil gaze movement in different directions turning upsidedown, in a way that let the computer to predict that George was confused. Then the e-learning systems started showing tips and hints about this part, and it helped him in answering the question by in order to complete this part of the module to proceed.

Figure 2: Use case scenario of an adaptive e-learning system.

\section{AFFECTIVE STATE PERCEPTION}

Generally speaking, 'Affect' as a concept describes the feeling that a human experiences during everyday activities (Stangor, Jhangiani and Hammond, 2014). Additionally, 'Affect' is a broad term that can be related to emotions, mood and any reaction yielded instinctively due to a response caused by specific incident or stimuli (Zajonc, 1980; Stangor, Jhangiani and Hammond, 2014).

Affective State (i.e. emotion) represents a composition of more complicated psychological and physiological constructs (Harmon-Jones, Gable and Price, 2012). Affective State is associated with perceptible biological changes, which causes the human to feel it, as well as the opportunity of the appearance of such changes that make them apparent for other humans so that they might observe the feeling that the person is experiencing (Davidson, 1992). Subsequently, Oatley and Johnson-laird described Affective States as cognitive states that coordinate nervous system processes, which make the biological changes to achieve certain goals through transition between different plans, together with communicating and conveying these goals to others (Oatley and Johnson-Laird, 1987).

Affective States have been studied by many cognitive psychologists because of the cause-effect relationship between emotional state and other 
cognitive processes, such as working memory, attention and reasoning operations (Zajonc, 1980; Oatley and Johnson-Laird, 1987; Harmon-Jones, Gable and Price, 2012). Affective State has a profound impact on the cognitive processes, brain activation and attention, for example the Happy emotional state strengthens cognition and broadens the cognitive scope, whereas the Anxiety emotional state has the opposite effect (Oatley and JohnsonLaird, 1987; Harmon-Jones, Gable and Price, 2012).

Subsequently, several theories have been developed by psychologists to explain the relationship between Affective State and cognitive abilities, in spite of the fact that many of these theories do not address the type of a particular Affective State within a specific context such as critical thinking or learning (Graesser et al., 2008).

For instance, Frijda and Parrott proposed the "Action-Readiness Theory of Emotions" that describes what they call UR-Emotions - that are multicomponent emotions - a set of stimulusresponse pairs, which are universal and based on the biological changes and represents states of readiness for specific actions (Frijda and Parrott, 2011).

Additionally, the most common employed theory for Affective State is the "Core-Affect Theory of Emotions" introduced by (Russell, 2003) and elaborated on by (Yik, Russell and Steiger, 2011). This theory advises that emotion is a state resulting from core affect and comprises a set of underlying dimensions as given in Figure 3, which composed of a Valence component that describes the pleasure level, and an Arousal component that refers to the activation level (Russell, 2003; Harmon-Jones, Gable and Price, 2012).

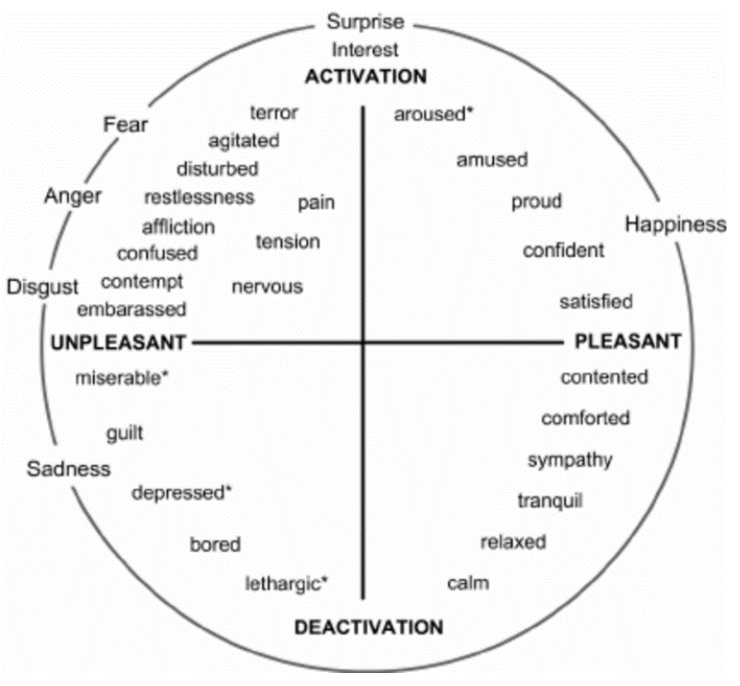

Figure 3: Valence (pleasant-unpleasant continuum) versus Arousal (activation-deactivation continuum). The two dimensional spaces of the Circumplex Model (Russell and Lemay, 2000).

\section{USER PERPLEXITY IN HUMAN-COMPUTER INTERACTION}

With regard to the adaptive $\mathrm{HCl}$ context, detecting the Affective State and information about the confusion of a user, or any emotional state that is directly related to the interactive task being undertaken which predicts an adverse impact on task completion. This is considered the most relevant information for the system in order to apply the appropriate adaptation before the user abandons the task.

Therefore, a novel terminology has been used in this paper that underlines that adaptive $\mathrm{HCl}$ should be able to detect the User Perplexity State. The term 'Perplexity' is literally defined in Oxford dictionary as "Inability to deal with or understand something" (Oxford English Dictionary, 2016). Moreover, 'Perplexity' is defined in the Britannica Encyclopaedia as "the state of being very confused because something is difficult to understand" (Encyclopedia Britannica, 2016). Furthermore, its definition in the Cambridge Dictionary is "a state of confusion or a complicated and difficult situation or thing" (Cambridge Dictionary, 2016). Thus, inferring User Perplexity in the $\mathrm{HCl}$ context is sensible given that confusion in Affective State detection is considerably important since it represents the actual entry point for artificial intelligence to facilitate adaptive $\mathrm{HCl}$ or UX (Graesser et al., 2008).

For this reason, an adaptive $\mathrm{HCl}$ has to be able to capture the episodes of when the user is perplexed whilst they are interacting with software. However, recognising User Perplexity and Confusion is still non-trivial and a challenging objective, particularly in the context of $\mathrm{HCl}$. Additionally, the complexity of human emotions and the peculiarity of the relationship between humans and machines provides a challenge in Affective Computing and relevant research themes (Samara et al., 2016, 2017).

\section{CONCLUSION}

This paper described self-aware and adaptive $\mathrm{HCl}$ systems together with use-case scenarios of affectaware systems, which infer the user's level of perplexity and respond by arranging a suitable form of support in order to permit task completion effectively. Furthermore, this paper presented the User Perplexity abstraction, which should provide details about the user's perplexity state, which should, in turn, necessitate appropriate assistance. Accordingly, User Perplexity represents the affective state that the adaptive $\mathrm{HCl}$ system shall be able to detect. 


\section{REFERENCES}

Busso, C. et al. (2004) 'Analysis of emotion recognition using facial expressions, speech and multimodal information', ... on Multimodal ..., pp. 205-211. doi: 10.1145/1027933.1027968.

Cambridge Dictionary, C. (2016) 'Cambridge Dictionary', Meaning, (entry 124), pp. 138-138.

Davidson, R. J. (1992) 'Anterior cerebral asymmetry and the nature of emotion', Brain and Cognition, 20(1), pp. 125-151. doi: 10.1016/02782626(92)90065-T.

Ekman, P. and Friesen, W. V (1971) 'Constants across cultures in the face and emotion.', Journal of personality and social psychology, 17(2), pp. 124129. doi: $10.1037 / \mathrm{h} 0030377$.

Encyclopedia Britannica (2016) 'Britannica Online Encyclopedia', Encyclopædia Britannica, pp. 1-73. Available at: http://www.britannica.com/.

Frijda, N. H. and Parrott, W. G. (2011) 'Basic emotions or ur-emotions?', Emotion Review. Sage Publications Sage UK: London, England, 3(4), pp. 406-415.

Graesser, A. C. et al. (2008) 'The relationship between affective states and dialog patterns during interactions with AutoTutor', Journal of Interactive Learning Research, 19(2), pp. 293-312.

Harmon-Jones, E., Gable, P. A. and Price, T. F. (2012) 'The influence of affective states varying in motivational intensity on cognitive scope', Frontiers in Integrative Neuroscience, 6. doi: 10.3389/fnint.2012.00073.

Harper, R. et al. (2008) Being Human - HumanComputer Interaction in the Year 2020, Cambridge University Press. doi: citeulike-article-id:3349429.

Karray, F. et al. (2008) 'Human-Computer Interaction: Overview on State of the Art', INTERNATIONAL JOURNAL ON SMART SENSING AND INTELLIGENT SYSTEMS, 1, pp. 137-159.

Liu, Z. and Baker, R. S. J. D. (2013) 'Sequences of Frustration and Confusion, and Learning', in Proceedings of the 6th International Conference on Educational Data Mining, pp. 114-120.

O'Brien, H. L. and Toms, E. G. (2008) 'What is user enagement? A Conceptual Framework for defining user engagement with technology', Journal of the American Society for Information Science and Technology, 59(6), pp. 938-955. doi: 10.1002/asi.20801.1.

Oatley, K. and Johnson-Laird, P. N. (1987) 'Towards a Cognitive Theory of Emotions', Cognition and Emotion, 1(1), pp. 29-50. doi: $10.1080 / 02699938708408362$.
Oxford English Dictionary (2016) Oxford English Dictionary Online, Oxford English Dictionary. Available at: http://dictionary.oed.com.

Picard, R. W. (2000) 'Perceptual user interfaces: affective perception', Communications of the ACM. ACM, 43(3), pp. 50-51.

Russell, J. A. (2003) 'Core affect annd the psychological construction of emotion', Psychological Review, 110(1), pp. 145-172. doi: 10.1037/0033-295X.110.1.145.

Russell, J. and Lemay, G. (2000) 'Emotion Concepts. Handbook of Emotion. MH-J. Lewis, M. New York'. Guilford Press.

Samara, A. et al. (2016) 'Sensing Affective States using Facial Expression Analysis', in Ubiquitous Computing and Ambient Intelligence. 10th International Conference, UCAmI 2016, San Bartolomé de Tirajana, Gran Canaria, Spain, November 29 - December 2, 2016, Proceedings, Part I. Springer International Publishing, pp. 341352. doi: 10.1007/978-3-319-48746-5_35.

Samara, A. et al. (2017) 'Affective state detection via facial expression analysis within a humancomputer interaction context', Journal of Ambient Intelligence and Humanized Computing. doi: 10.1007/s12652-017-0636-8.

Shan, C., Gong, S. and McOwan, P. W. (2007) 'Beyond Facial Expressions: Learning Human Emotion from Body Gestures', Procedings of the British Machine Vision Conference 2007, p. 43.143.10. doi: 10.5244/C.21.43.

Stangor, C., Jhangiani, R. and Hammond, T. (2014) Principles of social psychology. BC Campus.

Yik, M., Russell, J. A. and Steiger, J. H. (2011) 'A 12-Point Circumplex Structure of Core Affect', Emotion, 11(4), pp. 705-731. doi: 10.1037/a0023980.

Zajonc, R. B. (1980) 'Feeling and thinking: Preferences need no inferences', American Psychologist, 35(2), pp. 151-175. doi: 10.1037/0003-066X.35.2.151. 